\title{
Measurement of photon production at ATLAS *
}

\author{
Daniel Camarero
}

Departamento de Física Teórica C-15 and CIAFF, Universidad Autónoma de Madrid, Madrid, Spain on behalf of the ATLAS Collaboration ${ }^{1}$

\begin{abstract}
The production of isolated prompt photons at hadron colliders provides stringent tests of perturbative QCD and can be used to evaluate probability distribution functions of partons in the proton. At the LHC, the ATLAS Collaboration has performed precise measurements of the inclusive production of isolated-prompt photons in proton-proton collisions at a centre-of-mass energy of $13 \mathrm{TeV}$. The ratio of the cross sections for inclusive isolated-photon production at centre-of-mass energies of 13 and $8 \mathrm{TeV}$ has been measured as a function of the photon transverse energy in different regions of the photon pseudorapidity. The measurements are compared with state-of-the art theory predictions at nextto-leading as well as next-to-next-to-leading order in perturbative QCD in the case of the measurement of inclusive production of isolated-prompt photons.
\end{abstract}

Keywords: ATLAS, Standard Model, QCD, photon

\section{Introduction}

The production of prompt-photons with large transverse momenta constitutes a hard colourless probe of the hard interactions and its production in proton-proton collisions, $p p \rightarrow \gamma+X$, provides a testing ground for perturbative QCD (pQCD). Prompt photons are defined as those that are not secondaries from hadron decays. Since the dominant production mechanism in proton-proton collisions at the LHC proceeds via the $q g \rightarrow q \gamma$ process, measurements of prompt-photon production are sensitive to the gluon density in the proton. These measurements can also be used to tune Monte Carlo (MC) models to improve our understanding of prompt-photon production and aid those analyses for which events containing photons are an important background.

At leading order (LO) in pQCD, prompt-photon production is understood to proceed via two processes:

*Talk given at 22nd International Conference in Quantum Chromodynamics, 2 July - 5 July 2019, Montpellier - France

${ }^{1}$ Copyright 2019 CERN for the benefit of the ATLAS Collaboration. CC-BY-4.0 license. the direct-photon process, in which the photon originates directly from the hard interaction, and the fragmentation-photon process, in which the photon is emitted in the fragmentation of a high transverse momentum $\left(p_{\mathrm{T}}\right)$ parton.

Photons are reconstructed from clusters of energy deposited in the electromagnetic (EM) calorimeter and classified as unconverted (clusters without a matching track or without a matching reconstructed conversion vertex in the inner detector) or converted (clusters with a matching reconstructed conversion vertex or a matching track consistent with originating from a photon conversion). After reconstruction, the photon energy is calibrated. The calibration accounts for upstream energy loss as well as lateral and longitudinal leakages.

The main background in the prompt-photon production measurement comes from an energetic $\pi^{0}$ or $\eta$ meson which is misidentified as a photon because it decays into an almost collinear photon pair. Such energetic $\pi^{0}$ or $\eta$ mesons are produced copiously inside jets. This background is reduced by the photon identification criteria and by requiring the photon candidate to be isolated. Photon candidates are identified by using vari- 
ables to characterise the lateral and longitudinal electromagnetic shower development in the EM calorimeter and the energy fraction leaking into the hadronic calorimeter. Tight requirements are imposed on the shower shapes in the second layer and in the finely segmented first layer of the EM calorimeter as well as on the energy deposited in the hadronic calorimeter.

In proton-proton collisions, due to the abundance of photons from hadron decays and the contribution from the fragmentation process, prompt-photon production is studied by requiring the photons to be isolated. The photon isolation requirement is based on the amount of transverse energy $\left(E_{\mathrm{T}}^{\text {iso }}\right)$ inside a cone of size $\Delta R=0.4$ in the $\eta-\phi$ plane around the photon candidate, excluding an area of size $\Delta \eta \times \Delta \phi=0.125 \times 0.175$ centred on the barycentre of the photon cluster. The measured value of $E_{\mathrm{T}}^{\text {iso }}$ is computed from topological clusters of calorimeter cells and is corrected for the expected leakage of the photon energy into the isolation cone as well as for the contributions from the underlying event (UE) and pile-up. After the corrections, $E_{\mathrm{T}}^{\text {iso }}$ is required to be less than $E_{\mathrm{T} \text {,cut }}^{\text {iso }} \equiv 4.2 \times 10^{-3} \times E_{\mathrm{T}}^{\gamma}+4.8 \mathrm{GeV}$, where $E_{\mathrm{T}}^{\gamma}$ is the transverse energy of the photon. The same value of $E_{\mathrm{T} \text {,cut }}^{\text {iso }}$ is used in the two analyses presented.

The measurements of inclusive isolated-photon cross sections performed by ATLAS [1] at $13 \mathrm{TeV}$ [2] and $8 \mathrm{TeV}$ [3] were compared with the predictions of pQCD at next-to-leading order (NLO). At both centre-of-mass energies $(\sqrt{s})$, the uncertainties affecting the predictions are dominated by terms beyond NLO and are larger than those of experimental nature, preventing a more precise test of the theory. An avenue to reach a more stringent test of the theory is to perform the measurement of the ratio of cross sections for inclusive isolated-photon production at 13 and $8 \mathrm{TeV}\left(R_{13 / 8}^{\gamma}\right)$ and compare it with the prediction. The impact of the experimental systematic and theoretical uncertainties on the ratio of the cross sections is reduced by accounting for the correlations between the two $\sqrt{s}$, allowing a more precise comparison between data and theory. A further reduction of the experimental uncertainty can be achieved by measuring a double ratio of $R_{13 / 8}^{\gamma}$ to the ratio of the fiducial cross section for $Z$ boson production at 13 and $8 \mathrm{TeV}\left(R_{13 / 8}^{Z} \equiv \sigma_{Z}^{\mathrm{fid}}(13 \mathrm{TeV}) / \sigma_{Z}^{\mathrm{fid}}(8 \mathrm{TeV})\right)$ presented in [4], since the uncertainties from the luminosity measurement cancel out, and $D_{13 / 8}^{\gamma / Z}$ has only a slightly larger theoretical uncertainty than $R_{13 / 8}^{\gamma}$.

The NLO pQCD predictions of JETPHOX based on several parameterisations of the parton distribution functions (PDFs) are compared with the measurements performed in both analyses [5, 6]. For the inclusive isolated-photon measurement, the prediction of SHERPA is compared with the measurement as well as the recent next-to-next-to-leading order (NNLO) pQCD prediction of NNLOJET [7], which has significantly reduced uncertainties due to the inclusion of NNLO higher-order terms.

\section{Ratio of inclusive-photon cross sections at $\mathbf{1 3}$ and $8 \mathrm{TeV}$}

The measurement of $\mathrm{d} \sigma / \mathrm{d} E_{\mathrm{T}}^{\gamma}$ at $\sqrt{s}=8 \mathrm{TeV}$ (13 TeV) used in the measurement of $R_{13 / 8}^{\gamma}$ is based on an integrated luminosity of $20.2 \pm 0.4 \mathrm{fb}^{-1}$ $\left(3.16 \pm 0.07 \mathrm{fb}^{-1}\right)$. The measurement of $R_{13 / 8}^{\gamma}$ covers the range $E_{\mathrm{T}}^{\gamma}>125 \mathrm{GeV}$, and is performed separately in four regions of the photon pseudorapidity $\left(\eta^{\gamma}\right)$, namely $\left|\eta^{\gamma}\right|<0.6,0.6<\left|\eta^{\gamma}\right|<1.37,1.56<\left|\eta^{\gamma}\right|<1.81$ and $1.81<\left|\eta^{\gamma}\right|<2.37$.

\subsection{Experimental uncertainties}

The systematic uncertainties associated with the photon energy scale $(\gamma \mathrm{ES})$ and resolution represent the dominant experimental uncertainties of the differential cross sections for inclusive isolated-photon production at both $\sqrt{s}$. The uncertainty arising from the $\gamma \mathrm{ES}$ in $R_{13 / 8}^{\gamma}$ is estimated by decomposing it into uncorrelated sources for both the 8 and $13 \mathrm{TeV}$ measurements. All the uncertainties are taken as fully correlated except for those taking into account the differences in the configuration of the ATLAS detector between 2012 and 2015. The relative uncertainty due to the correlated components of the $\gamma \mathrm{ES}$ in $R_{13 / 8}^{\gamma}$ is shown in Figure 1 as a function of $E_{\mathrm{T}}^{\gamma}$ for the first $\left|\eta^{\gamma}\right|$ region. The result of estimating that part of the systematic uncertainty assuming no correlation is also shown. The results obtained using the full correlation model exhibit a large reduction in comparison to those in which the correlations are ignored, demonstrating that a proper treatment of the inter $-\sqrt{s}$ correlations in the systematic uncertainties associated with the $\gamma \mathrm{ES}$ is important. The relative uncertainty due to the uncorrelated components of the $\gamma \mathrm{ES}$ and the components specific to 2015 is also shown in Figure 1. The relative uncertainty in $R_{13 / 8}^{\gamma}$ due to the $\gamma \mathrm{ES}$ is no longer the dominant uncertainty, except for $E_{\mathrm{T}}^{\gamma}>300 \mathrm{GeV}$ in the regions $0.6<\left|\eta^{\gamma}\right|<1.37$ and $1.56<\left|\eta^{\gamma}\right|<1.81$.

The other sources of uncertainty are treated conservatively as uncorrelated due to the differences in the estimation methods, the re-optimisation of the photon identification requirements, the running conditions and the generator versions used for their estimation. The total relative systematic uncertainty in $R_{13 / 8}^{\gamma}$ is below $5 \%$ 


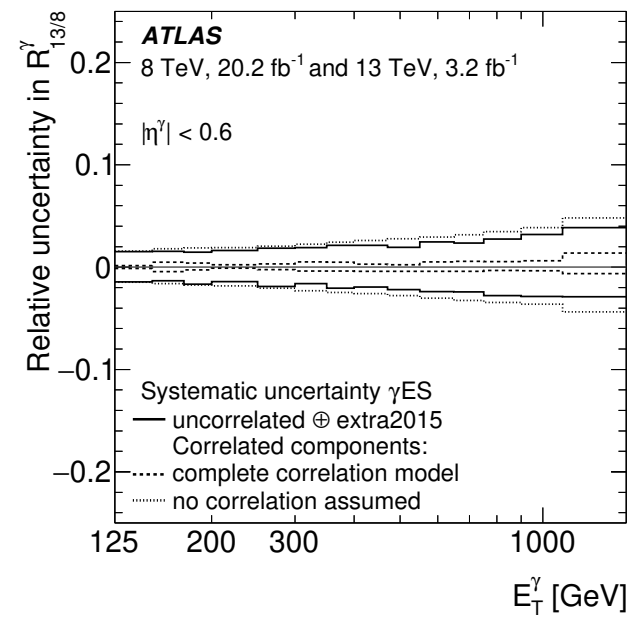

Figure 1: Relative systematic uncertainty in $R_{13 / 8}^{\gamma}$ as a function of $E_{\mathrm{T}}^{\gamma}$ in the first $\left|\eta^{\gamma}\right|$ region due to the $\gamma \mathrm{ES}$ correlated components (dashed lines). The results of considering the components as uncorrelated (dotted lines) and the uncertainty due to the uncorrelated components of the $\gamma \mathrm{ES}$ and those specific to 2015 (solid lines) are also shown [5].

for most of the phase-space considered, and is shown in Figure 2 as a function of $E_{\mathrm{T}}^{\gamma}$ for the first $\left|\eta^{\gamma}\right|$ region.

The total experimental uncertainty in $D_{13 / 8}^{\gamma / Z}$ is obtained adding in quadrature the uncertainty in $R_{13 / 8}^{\gamma}$ (not including the contribution of the luminosity) to the statistical $(0.1 \%)$ and systematic $(0.7 \%)$ uncertainties in $R_{13 / 8}^{Z}$. A comparison between the total relative systematic uncertainties in $R_{13 / 8}^{\gamma}$ and $D_{13 / 8}^{\gamma / Z}$ is provided in Figure 2 . The cancellation of the luminosity uncertainty in $D_{13 / 8}^{\gamma / Z}$ has a significant impact except at high $E_{\mathrm{T}}^{\gamma}$, where the statistical uncertainty dominates.

\subsection{Theoretical predictions}

The theoretical prediction for $R_{13 / 8}^{\gamma}$ is based on the NLO pQCD calculation computed using the program JETPHOX 1.3.1_2. This program provides NLO QCD calculations of direct and fragmentation contributions to the prompt-photon cross section. The renormalisation $\left(\mu_{\mathrm{R}}\right)$, factorisation $\left(\mu_{\mathrm{F}}\right)$ and fragmentation $\left(\mu_{\mathrm{f}}\right)$ scales are chosen to be $\mu_{\mathrm{R}}=\mu_{\mathrm{F}}=\mu_{\mathrm{f}}=E_{\mathrm{T}}^{\gamma}$. A fixed-cone isolation with $\Delta R=0.4$ is applied at parton level. Predictions are based on several PDF sets, namely MMHT2014, CT14, ABMP16, HERAPDF2.0 and NNPDF3.0.

Correction factors for the non-perturbative (NP) effects of hadronisation and the UE are estimated with PYTHIA and applied to the ratio prediction of JETPHOX. The uncertainties due to the scale variations, the proton PDFs, $\alpha_{\mathrm{s}}$, the impact of the beam energy uncertainty

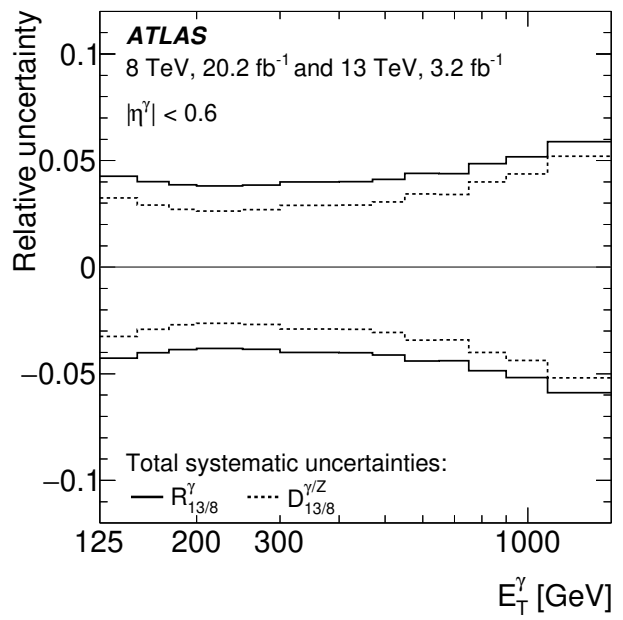

Figure 2: Relative total systematic uncertainties in $R_{13 / 8}^{\gamma}$ (solid lines) and $D_{13 / 8}^{\gamma / Z}$ (dashed lines) as functions of $E_{\mathrm{T}}^{\gamma}$ in the first $\left|\eta^{\gamma}\right|$ region [5].

and NP effects are evaluated. The dominant theoretical uncertainty arises from the variation of the scales.

To estimate the theoretical uncertainty in $R_{13 / 8}^{\gamma}$, it is crucial to consider the correlation between the two $\sqrt{s}$. The uncertainties due to the PDFs, $\alpha_{\mathrm{s}}$, beam energy and NP effects are fully correlated between the two $\sqrt{s}$. However, for the scale uncertainties the correlation is a priori unknown. In the standard approach, varying the scales coherently or incoherently at both $\sqrt{s}$ leads to very different theoretical uncertainties. In the coherent case, there are large cancellations in the uncertainties of the prediction of $R_{13 / 8}^{\gamma}$. The envelope of the scale variations is below $2 \%$ across most of the range in $E_{\mathrm{T}}^{\gamma}$, whereas in incoherent case, it is $O(14 \%)$ in all regions of phase space. A second approach free from ambiguity in the correlation is investigated computing the uncertainty as the difference between the LO and NLO predictions for $R_{13 / 8}^{\gamma}$. The differences found are up to $3.5 \%$, similar to the estimate based on the standard approach with a coherent variation of the scales and, thus, supporting its choice. The total relative uncertainty is below $2 \%(4 \%)$ at low (high) $E_{\mathrm{T}}^{\gamma}$ in all regions of $\left|\eta^{\gamma}\right|$.

The theoretical prediction for $D_{13 / 8}^{\gamma / Z}$ is based on NNLO pQCD calculations for the prediction of $R_{13 / 8}^{Z}$ using DYTURBO, and NLO pQCD calculations for the prediction of $R_{13 / 8}^{\gamma}$ using JETPHOX with the procedure described in the first paragraph of Section 2.2. The calculations using DYTURBO are based on several sets of PDFs, extracted using NNLO QCD fits, namely MMHT2014nnlo, CT14nnlo, HERAPDF2.0nnlo and NNPDF3.0nnlo. The uncertainties in the prediction of 
$R_{13 / 8}^{Z}$ due to the scale variations, the PDFs and $\alpha_{\mathrm{s}}$ are ${ }_{-0.3}^{+0.02} \%,{ }_{-0.8}^{+0.9} \%$ and ${ }_{-0.3}^{-0.03} \%$ respectively. For the $D_{13 / 8}^{\gamma / Z}$, the uncertainties are estimated considering the scale variations (PDF and $\alpha_{\mathrm{s}}$ ) as uncorrelated (fully correlated) between $Z$ boson production and isolated-photon production. The total relative uncertainty is below 3\% (4.5\%) at low (high) $E_{\mathrm{T}}^{\gamma}$ in all regions of $\left|\eta^{\gamma}\right|$.

\subsection{Results}

The measured $R_{13 / 8}^{\gamma}\left(D_{13 / 8}^{\gamma / Z}\right)$ as a function of $E_{\mathrm{T}}^{\gamma}$ in the first region of $\left|\eta^{\gamma}\right|$ is shown in Figure 3 (4). The measured $R_{13 / 8}^{\gamma}\left(D_{13 / 8}^{\gamma / Z}\right)$ increases with $E_{\mathrm{T}}^{\gamma}$ from approximately 2 (1.4) at $E_{\mathrm{T}}^{\gamma}=125 \mathrm{GeV}$ to approximately 8-29 (5-19) at the high end of the spectrum. In the forward regions, the increase of $R_{13 / 8}^{\gamma}\left(D_{13 / 8}^{\gamma / Z}\right)$ with $E_{\mathrm{T}}^{\gamma}$ is larger than in the central regions. At a fixed value of $E_{\mathrm{T}}^{\gamma}$, the measured $R_{13 / 8}^{\gamma}\left(D_{13 / 8}^{\gamma / Z}\right)$ increases with $\left|\eta^{\gamma}\right|$.

The significant reduction of the theoretical and experimental uncertainties obtained in $R_{13 / 8}^{\gamma}$ with respect to that obtained for the individual cross sections allows a more stringent test of NLO pQCD. The level of agreement between the data and NLO pQCD predictions based on several PDF sets within reduced uncertainties validates the description of the evolution of isolated-photon production in $p p$ collisions with $\sqrt{s}$.

\section{Measurement of inclusive-photon production at $13 \mathrm{TeV}$ with $36 \mathrm{fb}^{-1}$}

The differential cross section for isolated-photon production in $p p$ collisions is measured at $\sqrt{s}=13 \mathrm{TeV}$ with the ATLAS detector at the LHC using an integrated luminosity of $36.1 \mathrm{fb}^{-1}$ [6]. The differential cross section as a function of $E_{\mathrm{T}}^{\gamma}$ is measured for photons with $E_{\mathrm{T}}^{\gamma}>125 \mathrm{GeV}$ in the same regions of $\left|\eta^{\gamma}\right|$ defined in the first paragraph of Section 2.

\subsection{Systematic uncertainties}

Several sources of systematic uncertainties that affect the measurement are studied. The dominant systematic uncertainties arise from: the $\gamma \mathrm{ES}$, with uncertainties in the measured cross section in the range $1 \%-16 \%$; the photon identification efficiency, with uncertainties in the measured cross section below $2 \%$ except for $E_{\mathrm{T}}^{\gamma}>500 \mathrm{GeV}$ in the regions $0.6<\left|\eta^{\gamma}\right|<1.37$ and $1.56<\left|\eta^{\gamma}\right|<1.81$, where it increases to $3 \%$ and the integrated luminosity, which is $2.1 \%$. The total systematic uncertainty is in the range $3 \%-17 \%$, depending on $E_{\mathrm{T}}^{\gamma}$ and the $\left|\eta^{\gamma}\right|$ region.

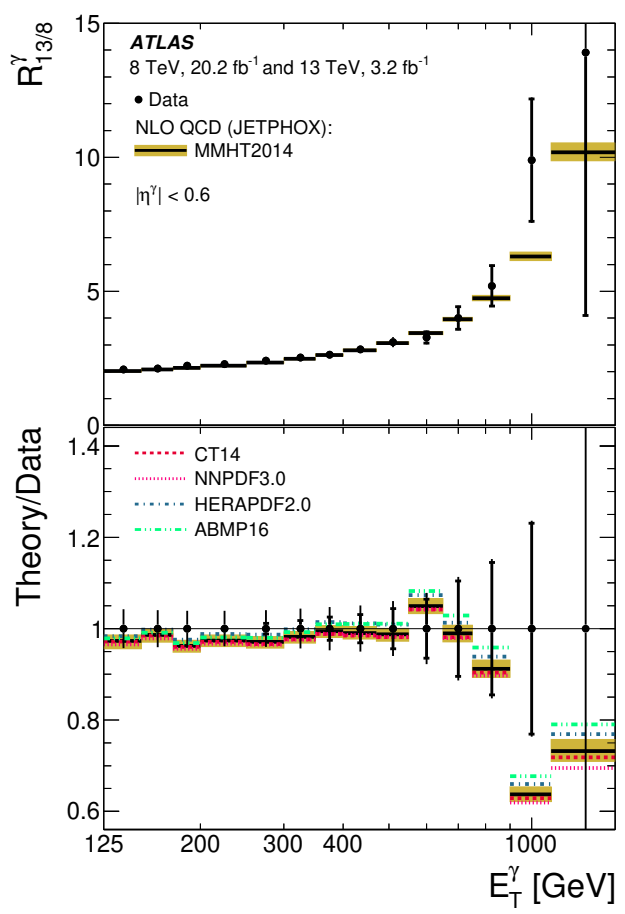

Figure 3: The measured $R_{13 / 8}^{\gamma}$ as a function of $E_{\mathrm{T}}^{\gamma}$ in the first $\left|\eta^{\gamma}\right|$ region. The NLO pQCD predictions of JETPHOX based on different PDF sets are also shown [5]

\subsection{Theoretical predictions}

The NLO pQCD predictions are computed using two programs, namely JETPHOX 1.3.1_2 and SHERPA 2.2.2. The JETPHOX program provides NLO pQCD calculations using the same procedure described in the first paragraph of Section 2.2. The SHERPA program consistently combines parton-level calculations of $\gamma+(1,2)-$ jet events at NLO in pQCD and $\gamma+(3,4)-$ jet events at LO, supplemented with a parton shower. Dynamic factorisation and renormalisation scales are adopted $\left(E_{\mathrm{T}}^{\gamma}\right)$, as well as a dynamical merging scale with $\bar{Q}_{\text {cut }}=20 \mathrm{GeV}$. The NNLO NNPDF3.0 PDF set is used. Photon isolation is applied at matrix-element by using Frixione's isolation and a fixed-cone at $\Delta R=0.4$ at particle level. The prescription employed is referred to as 'hybrid-cone isolation'.

The NNLO pQCD prediction is calculated in the NNLOJET framework. Only direct-photon processes are included; fragmentation processes are circumvented by the application of the Frixione's criterion at small $\Delta R$. The choice of the scales is $\mu_{\mathrm{R}}=\mu_{\mathrm{F}}=E_{\mathrm{T}}^{\gamma}$. The NNPDF3.1 PDF set at NNLO is used. Photon isolation is implemented following the 'hybrid-cone isolation' prescription. 


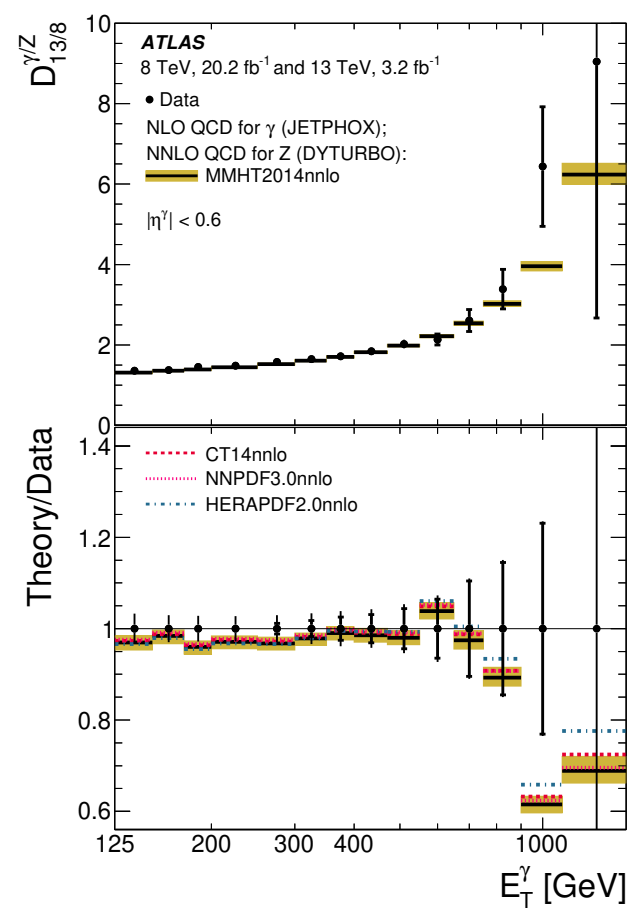

Figure 4: The measured $D_{13 / 8}^{\gamma / Z}$ as a function of $E_{\mathrm{T}}^{\gamma}$ in the first $\left|\eta^{\gamma}\right|$ region. The $\mathrm{pQCD}$ predictions based on different PDF sets are also shown [5].

For the JETPHOX and SHERPA predictions at NLO in QCD, the uncertainties due to the scale variations, the proton PDFs and $\alpha_{\mathrm{s}}$ are evaluated. Since the NLO prediction of JETPHOX is at the parton level, NP corrections are needed, as explained in Section 2.2. The corrections evaluated with PYTHIA are consistent with unity within $\pm 1 \%$. Thus, no correction is applied and an uncertainty of $1 \%$ is assigned. The dominant theoretical uncertainty for the prediction using JETPHOX (SHERPA) is that arising from the variation of the scales, which is in the range $10 \%-15 \%(20 \%-30 \%)$.

The uncertainties in the NNLO pQCD prediction arising from the scale variations are in the range $0.6 \%-$ $5 \%$ and are smaller than those in the NLO pQCD prediction by a factor in the range $2-20$, depending on $E_{\mathrm{T}}^{\gamma}$ and $\left|\eta^{\gamma}\right|$. The uncertainties due to the PDFs and $\alpha_{\mathrm{s}}$ are not available for the NNLO pQCD prediction, and as an approximation, those estimated with JETPHOX at NLO are taken for the calculation of the total uncertainty.

\subsection{Results}

The measured differential cross section and the NLO pQCD predictions of JETPHOX and SHERPA are compared in Figure 5 as functions of $E_{\mathrm{T}}^{\gamma}$ in different regions

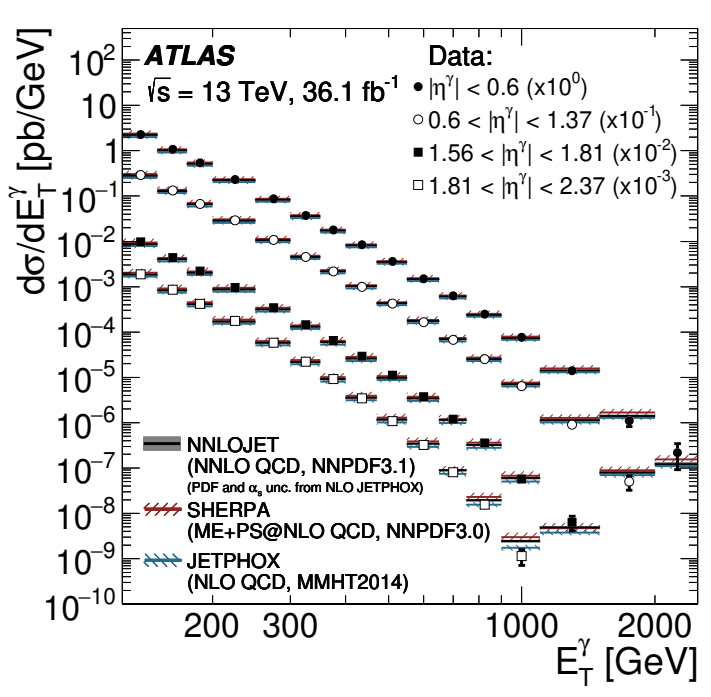

Figure 5: The measured differential cross section for isolated-photon production as a function of $E_{\mathrm{T}}^{\gamma}$ in different $\left|\eta^{\gamma}\right|$ regions. NLO pQCD predictions from JETPHOX and SHERPA and the NNLO pQCD predictions of NNLOJET are also shown [6].

of $\left|\eta^{\gamma}\right|$.

The ratios of the predictions of JETPHOX using different PDF sets to the measured cross section as a function of $E_{\mathrm{T}}^{\gamma}$ are shown in Figure 6. The prediction based on the MMHT2014 PDF set is closest to the data for $\left|\eta^{\gamma}\right|<1.37$ for most of the range in $E_{\mathrm{T}}^{\gamma}$. For $1.56<\left|\eta^{\gamma}\right|<2.37$, the prediction based on HERAPDF2.0 PDF set is closest to the data. The prediction based on the ABMP16 PDF set is further away from the data than that based on the MMHT2014 PDF set for $E_{\mathrm{T}}^{\gamma} \lesssim 1 \mathrm{TeV}$ in the region $\left|\eta^{\gamma}\right|<1.37$. The ratio of the prediction of SHERPA to the measured cross section is shown in Figure 7.

The inclusion of NNLO QCD corrections increases the normalisation of the prediction and reduces significantly the uncertainties due to the scale variations. This improvement is shown Figure 8 with the comparison of the NNLO and NLO pQCD predictions of NNLOJET.

\section{Summary and conclusions}

The ratio of cross sections for inclusive isolatedphoton production at $\sqrt{s}=13$ and $8 \mathrm{TeV}\left(R_{13 / 8}^{\gamma}\right)$ and the inclusive isolated-photon cross section at $\sqrt{s}=13 \mathrm{TeV}$ using an integrated luminosity of 36.1 $\mathrm{fb}^{-1}$ are measured in $p p$ collisions using data collected by the ATLAS detector at the LHC.

The measurement of the ratios $R_{13 / 8}^{\gamma}$ and $D_{13 / 8}^{\gamma / Z}$ constitutes a successful stringent test of the Standard Model 


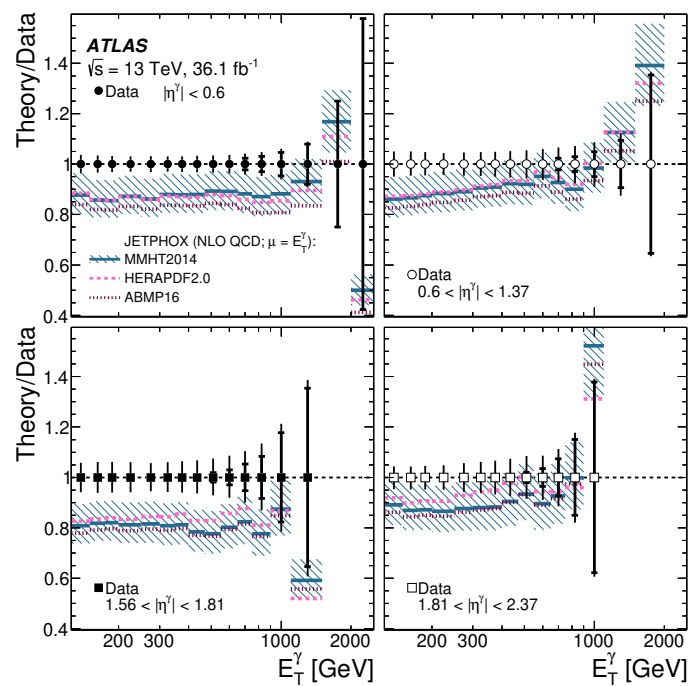

Figure 6: The ratio of the NLO pQCD predictions of JETPHOX using different PDF sets to the data as functions of $E_{\mathrm{T}}^{\gamma}$ in different $\left|\eta^{\gamma}\right|$ regions [6].

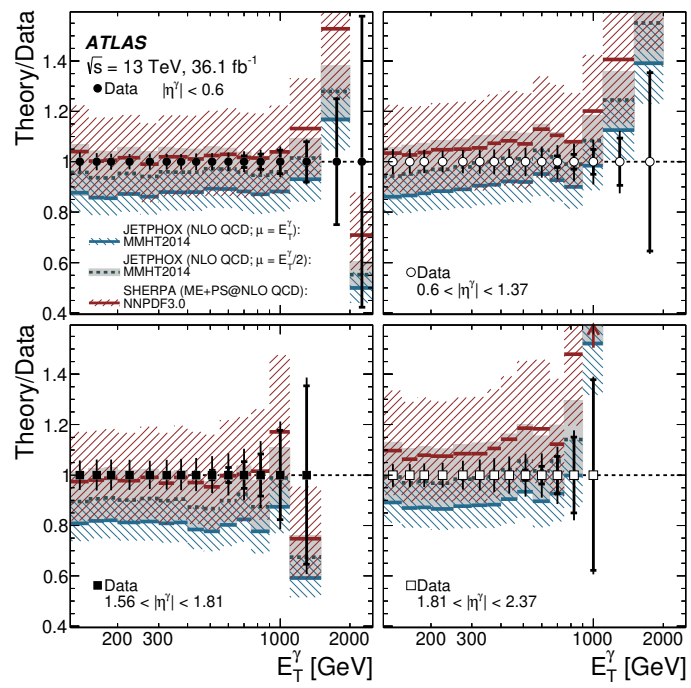

Figure 7: The ratio of the NLO pQCD predictions of JETPHOX with two different choices of the scales and SHERPA to the data as functions of $E_{\mathrm{T}}^{\gamma}$ in different $\left|\eta^{\gamma}\right|$ regions [6].

prediction thanks to the significant reduction of the experimental and theoretical uncertainties, dominated by the reduction of the $\gamma \mathrm{ES}$ systematic uncertainty and the uncertainty due to the terms beyond NLO respectively. The level of agreement between the NLO pQCD predition of JETPHOX based on different PDF sets and the data validates the description of the evolution of isolatedphoton production in $p p$ collisions from $\sqrt{s}=8$ to $13 \mathrm{TeV}$. The measurement of $D_{13 / 8}^{\gamma / Z}$ leads to a more

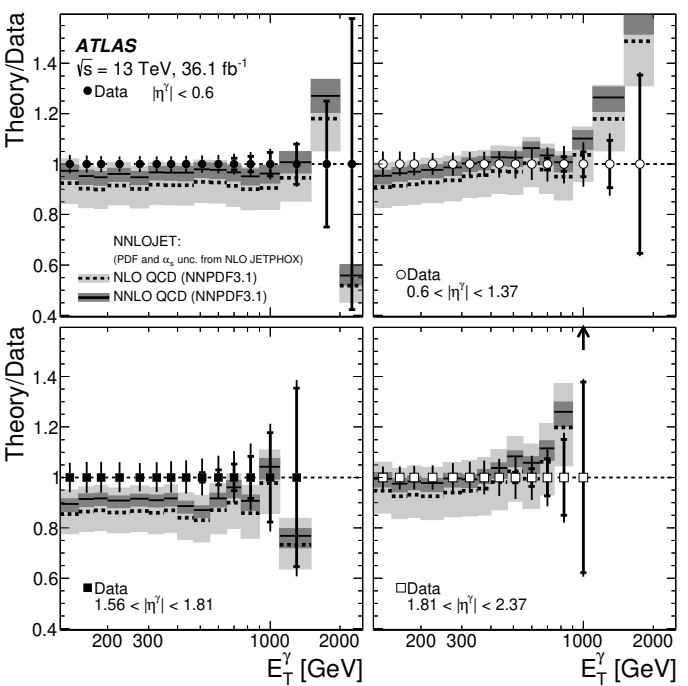

Figure 8: The ratio of the NLO and NNLO pQCD predictions of NNLOJET to the data as functions of $E_{\mathrm{T}}^{\gamma}$ in different $\left|\eta^{\gamma}\right|$ regions [6].

precise determination of the evolution of the inclusivephoton cross section with $\sqrt{s}$ normalised to the evolution of the $Z$ boson cross section.

The measurement of the inclusive isolated-photon represents an improvement in terms of statistical and systematic uncertainties relative to that published earlier thanks to the ten-fold increase in the integrated luminosity. The range $125<E_{\mathrm{T}}^{\gamma}<2500 \mathrm{GeV}$ is covered, extending the reach in $E_{\mathrm{T}}^{\gamma}$. The range in which the measurement is limited by the systematic uncertainties is extended upwards to $1 \mathrm{TeV}$ for $\left|\eta^{\gamma}\right|<1.37$. NLO pQCD predictions using several PDF sets are compared with the measurement and found to provide an adequate description of the data within the experimental and theoretical uncertainties. The comparison of the recently calculated NNLO pQCD prediction with the measured cross section represents a precise test of the theory at $O\left(\alpha_{\mathrm{EM}} \alpha_{\mathrm{s}}^{3}\right)$. The measurement has the potential to further constrain the PDFs, particularly the gluon density in the proton, within a global NNLO QCD fit.

\section{References}

[1] ATLAS Collaboration, 2008 JINST 3 S08003.

[2] ATLAS Collaboration, Phys. Lett. B 770 (2017) 473.

[3] ATLAS Collaboration, JHEP 08 (2016) 005.

[4] ATLAS Collaboration, JHEP 02 (2017) 117.

[5] ATLAS Collaboration, JHEP 04 (2019) 093.

[6] ATLAS Collaboration, arXiv:1908.02746 [hep-ex].

[7] X. Chen et al., arXiv:1904.01044 [hep-ph]. 